\title{
HIGH RESOLUTION OBSERVATIONS OF THE SOLAR CORONA
}

\author{
D. GOMEZ† AND L. GOLUB \\ IIarvard-Smithsonian Center for Astrophysics \\ 60 Garden St., Cambridge, MA 02138, USA
}

Soft X-ray images of the solar corona obtained during the last 20 years have systematically shown an intimate relationship between intense emitting structures and magnetic fields (Vaiana and Rosner 1978). The magnetic field confines a $10^{6} \mathrm{~K}$ plasma, which is an intense source of soft X-ray photons. Therefore, it is natural to expect the bright X-ray structures to follow the field's geometry. But this relationship does not seem to be just geometrical. It is generally believed that the energy necessary to heat the plasma comes from the dissipation of magnetic stresses, which are continually being re-generated by subphotospheric convective motions. However, there is still great uncertainty about the precise physical mechanisms involved in the production and release of the magnetic energy.

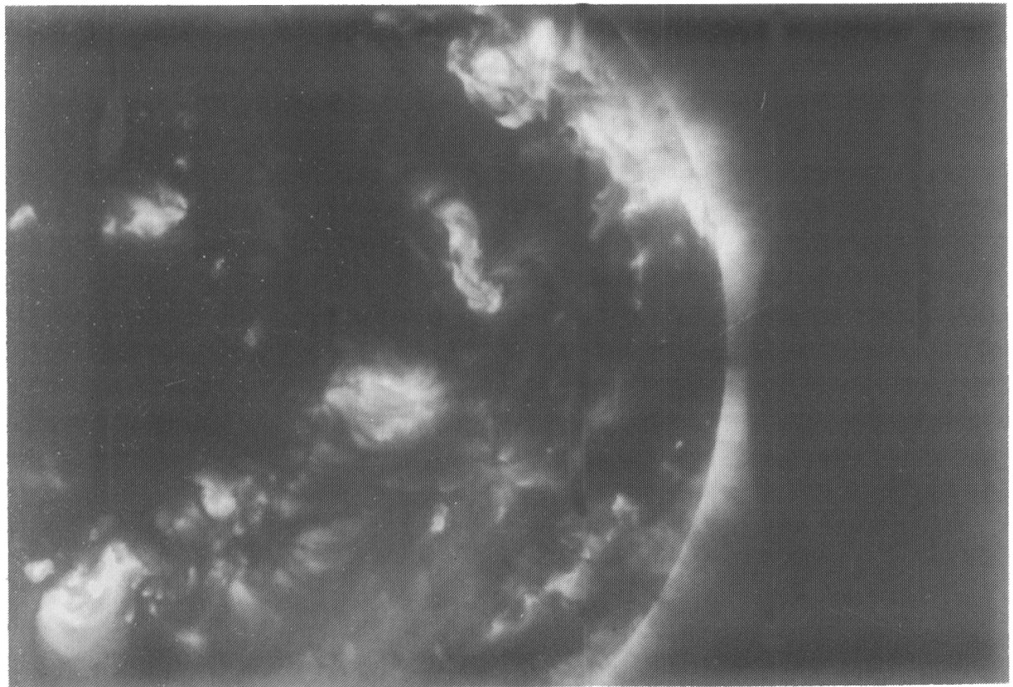

Figure 1. X-ray image of the solar corona obtained by NIXT, $11 \mathrm{July}$ 1991, 17:30 UT, showing the highly filamentary structure of coronal active regions.

The Normal Incidence X-ray Telescope (NIXT) has recently obtained very high spatial resolution images of the solar corona in a series of successful sounding rocket flights (see

$\dagger$ Present address: Institute for Astronomy, University of Hawaii, 2680 Woodlawn Dr., Honolulu, HI 96822, USA

$$
659
$$

J. Bergeron (ed.), Highlights of Astronomy, Vol. 9, 659-660.

(C) 1992 IAU. Printed in the Netherlands. 
Figure 1). A close inspection of these observations along with simultaneous magnetograms, confirms the general picture described above. However, because of their comparatively higher spatial resolution ( 0.75 arcsec.), much finer structuring as well as a wealth of new features have also been observed (Golub et al. 1990).

NIXT was configured as a $25 \mathrm{~cm}$. diameter, $\mathrm{f} / 8$ prime focus instrument. It uses multilayer coatings on its mirrors to increase the $X$-ray reflectivity, allowing the imaging of Fe XVI and Mg X coronal emission lines near a wavelength of $63.5 \stackrel{\circ}{A}$ and a passband of $1.4 \stackrel{A}{ }$ at FWHM (Golub and Herant 1989).

The coronal loops observed by NIX'T show a highly fibrilated internal structure, all the way down to the resolution limit of the instrument $(600 \mathrm{~km}$ on the solar surface). Therefore, it seems natural to speculate that still finer scale structures will be seen on improved spatial resolution observations. Since most of the models of coronal heating require the development of microscopic dissipative structures where magnetic energy is efficiently released (for a review on coronal heating theories, see Narain and Ulmschneider 1990; also Gómez 1990), the observational confirmation of this assertion will have important consequences on theoretical models. These dissipative structures are nothing but regions where the magnetic field is strongly sheared, like the stochastic distribution of current sheets proposed by Parker (1972) or the tangential discontinuities where shear Alfven waves can be dissipated (Heyvaerts and Priest 1983). The presence of a stationary regime of MIID turbulence can naturally explain the formation of dissipative structures (van Ballegooijen 1986, Gómez and Ferro Fontán 1988). We therefore searched for observational indications of a turbulent regime. A Fourier analysis over a number of different active regions strongly suggests this possibility (Gómez et al. 1991).

Comparison of X-ray images with $H_{\alpha}$ brightenings at the footpoints of the loops, show that coronal loops near sunspots preferentially end in the spot penumbrae. No hot loops are found terminating in sunspot umbrae. This observational result indicates that the subphotospheric driving of magnetic stresses is strongly inhibited in regions of intense magnetic fields, like sunspot umbrae.

Several flaring events have been observed by NIXT. Because NIXT response is lower at high temperatures, we are able to observe both the flare and the surrounding fainter regions in the same image (Golub 1991). Among the other striking new phenomena which were observed during these impulsive events, we note the presence of X-ray structures oriented along and within $H_{\alpha}$ ribbons. These structures are due to the existence of an involved magnetic topology at the arcsecond resolution level, which could not be resolved by previous X-ray observations.

\section{References}

Golub, L. 1991, in Mechanisms of Chromospheric and Coronal Heating (P. Ulmschneider, E.R. Priest, and R. Rosner eds.), Springer-Verlag, Heidelberg, p. 115.

Golub, L., and 7 co-authors 1990, Nature, 344, 842.

Golub, L., and Herant, M. 1989, Proc. SPIE, 1160, 629.

Gómez, D., and Ferro Fontán, C. 1988, Solar Phys., 116, 33.

Gómez, D. 1990, Fund. Cosmic Phys., 14, 131.

Gómez, D., Martens, P.C.H., and Golub, L. 1991, Astrophys. J., submitted.

Heyvaerts, J., and Priest, E.R. 1983, Astr. Ap., 137, 63.

Narain, U., and Ulmschneider, P. 1990, Space Sci. Rev., 54, 377.

Parker, E.N. 1972, Astrophys. J., 174, 499.

Vaiana, G., and Rosner, R. 1978, Ann. Rev. Astr. Ap., 16, 393.

van Ballegooijen, A.A. 1986, Astrophys. J., 311, 1001. 